\title{
A Lagrangian Method for Extracting Eddy Boundaries in the Red Sea and the Gulf of Aden
}

\author{
Anke Friederici * Habib Toye Mahamadou Kele ${ }^{\dagger} \quad$ Ibrahim Hoteit $^{\dagger}$ \\ Tino Weinkauf * Holger Theisel ${ }^{\ddagger} \quad$ Markus Hadwiger ${ }^{\dagger}$
}

\begin{abstract}
Mesoscale ocean eddies play a major role for both the intermixing of water and the transport of biological mass. This makes the identification and tracking of their shape, location and deformation over time highly important for a number of applications. While eddies maintain a roughly circular shape in the free ocean, the narrow basins of the Red Sea and Gulf of Aden lead to the formation of irregular eddy shapes that existing methods struggle to identify. We propose the following model: Inside an eddy, particles rotate around a common core and thereby remain at a constant distance under a certain parametrization. The transition to the more unpredictable flow on the outside can thus be identified as the eddy boundary. We apply this algorithm on a realistic simulation of the Red Sea circulation, where we are able to identify the shape of irregular eddies robustly and more coherently than previous methods. We visualize the eddies as tubes in space-time to enable the analysis of their movement and deformation over several weeks.
\end{abstract}

Index Terms: Flow visualization-Mesoscale eddies-Red SeaGulf of Aden-Vortex area-Lagrangian Boundaries

\section{INTRODUCTION}

Ocean eddies are bowl-shaped bodies of water that rotate around a vertical axis. They contribute to the energy conversions between largescale circulation and mesoscale dynamics, and regulate the bio-geochemical ocean's global cycle [21]. Most notably, they transport waters of a certain properties, like temperature and trace concentrations.

To support an analysis of eddy properties, a robust detection of their area is required. In the open ocean, their horizontal shape is circular, and the structure may remain stable for months. Several algorithms exist to identify and track them. The standard in the oceanography community is the local Okubo-Weiss criterion [16,23]. Another good indicator of eddy boundaries are Lagrangian Coherent Structures (LCS).

Eddies in the Red Sea region differ from their long-lived counterparts. The narrowness of the Red Sea exposes them to high strain. This causes the shape to deviate considerably from a circle and restricts the lifetime of Red Sea eddies to about two weeks [28]. These characteristics pose significant challenges to the above mentioned extraction algorithms.

To overcome these obstacles in this specific application, we present an algorithm for a reliable extraction and tracking. Given a vortex core line in the center of a Red Sea eddy, we analyze the behavior of particles circulating around it. Our goal is to distinguish between particles remaining near the vortex core over longer integration times, and particles drifting away from it. To this end, we re-parameterize the corresponding path lines based on their angular position, which allows for a meaningful comparison of lines with different angular speeds. Then, we integrate over the distance they build up to their neighbors

*KTH Royal Institute of Technology: \{ankef, weinkauf $\}$ kth.se

$\dagger$ King Abdullah University of Science and Technology (KAUST): \{habib.toyemahamadoukele, ibrahim.hoteit, markus.hadwiger\}@ kaust.edu.sa

${ }^{\ddagger}$ University of Magdeburg: theisel@ovgu.de over time. This provides a clear boundary between particles that rotate jointly around a core line and those that diverge. We apply our method on two velocity time series generated by a simulation of the Red Sea.

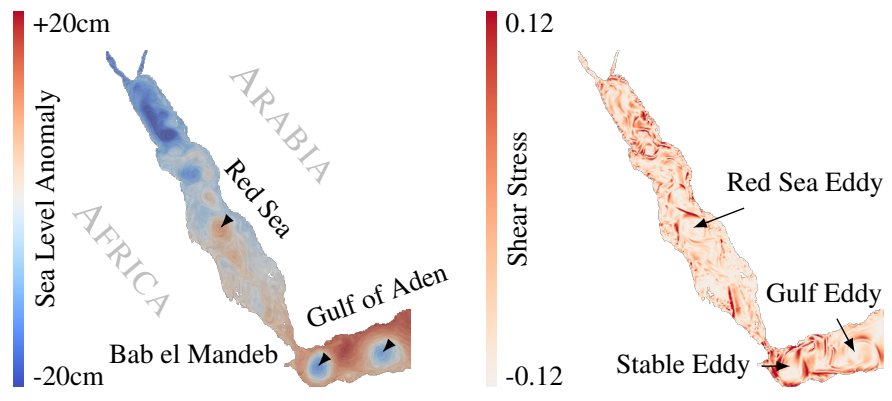

Figure 1: The Red Sea dataset. Geographical structures are annotated. a) Velocity field presented by a LIC, overlaid by color coded SLA. The three eddies appear as extremal areas. Several other vortical areas with slight surface level deviations disappear within less than a week.

b) Shear field. Areas of high shear overlap with eddy SLA regions.

\subsection{Eddy Properties}

The presence of an eddy can be observed in several ocean properties. At first glance, most eddies are easily made visible by the sea level anomaly (SLA), i.e., the variation of the water surface height from the mean measured over several decades. Depending on their rotation, eddies will appear as domes or bowls. As eddies transport water, the distribution of temperature, salinity and other tracers can be an indication of eddies, too. Another approach is to quantify the transport of mass directly, since an eddy is supposed to contain a relatively stable mass with little exchange across its boundary. This also means that water inside an eddy remains in circular rotation. Finally, eddies feature high vorticity, which quantifies rotating movement, and are usually surrounded by a ring of high shear where interacting with their surroundings.

\subsection{Red Sea and Gulf of Aden}

The Red Sea is a long and narrow basin that only connects to the open ocean through the shallow strait of Bab el Mandeb in the south. Directly behind this strait, water intermixes within the very narrow Gulf of Aden, which is part of the Arabian Sea and thus the Indian Ocean. Figure 1 provides a geographical overview of the region. Due to this shape, most eddies cover half or more of its width, providing rapid transport of organisms and nutrients between the coasts $[25,28]$.

Understanding the statistical properties and variability of eddies in the Red Sea will not only serve to improve local ocean forecasts but would also lead to greater understanding of the important physicalbiological and air-sea interactions in its basin [18]. The first step is a stable extraction of the eddies' area and their evolution over time.

\subsection{Dataset}

We analyze the output of a general circulation model that has been recently implemented and validated for the Red Sea and Gulf of Aden $[26,27]$. The dataset is shown in Figure 1. We have run the simulation on $\left(0.04^{\circ}\right)^{2}$ sized grid cells and a temporal resolution of 6 hours. The output dataset contains $500 \times 500$ cells over 32 days, totaling 128 time steps. Different initial conditions generated 10 similar instances.

With the SLA field, we identify the locations of three eddies. Other extrema are not regarded as their lifetime is shorter than one week. 


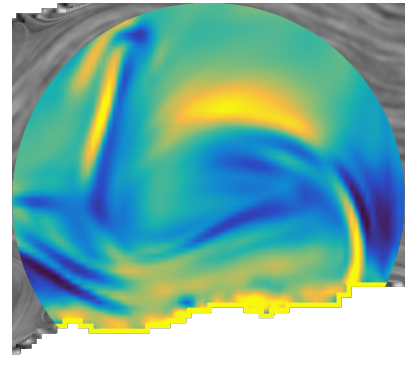

(a) Shear Strain

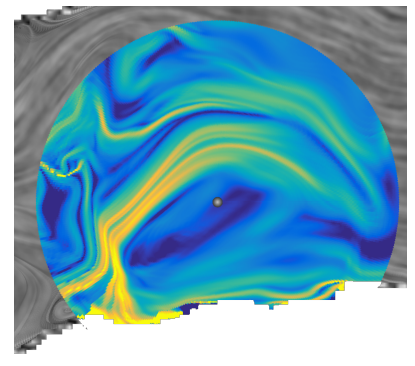

(b) Forward FTLE

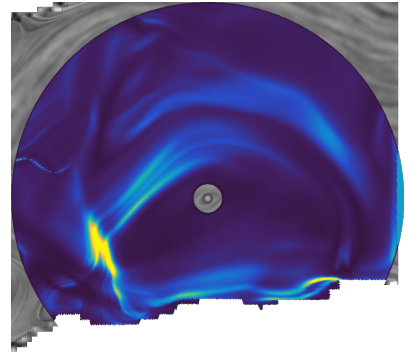

(c) Euclidean path line distance

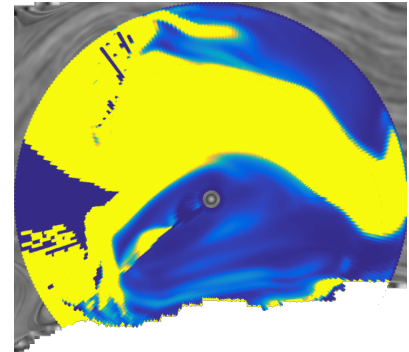

(d) Core distance error $E_{c}$

Figure 2: Comparison of distance-based methods. Integration times are set to 40 time steps in each case, the core position is marked. a) Shear strain values $S_{\text {shear }}$. b) Forward FTLE. The ridges mark fragments of the eddy boundary, but are difficult to extract. c) Euclidean distance between path lines over time. d) Our method: Error of distance over angle $\left(E_{c}\right)$. The clear cut borders are similar to the FTLE ridges but considerably better suited for a shape extraction.

\section{Related Work}

\section{Local Eddy Detection}

The simplest approach to identify eddies is by thresholding the sea level anomaly (SLA). A framework to analyze the uncertain SLA field of the Red Sea has been implemented by Höllt et al. [11,12]. While this method is useful to obtain a general idea of position and size, no exact boundary can be extracted with this alone.

Treating an eddy as a general vortex allows to apply standard vortex extractors. Early works applied a thresholding of vorticity alone [10], or set it in relation to the strain of the field [14]. Both vorticity $\Omega$, strain $S$ and shear strain $s_{\text {shear }}$ can be computed directly from the local Jacobian $J$. The most popular local method based on strain and vorticity in the geophysics community is the $R^{2}$ criterion, also known as the OkuboWeiss criterion [16,23]. Locally, $f_{O W}(x, y)=\|S\|^{2}-\|\Omega\|^{2}$ is evaluated. Regions fulfilling $f_{O W}<=-0.2 \times \sigma_{O W}$ are classified as eddies, with $\sigma_{O W}$ the standard deviation of $f_{O W}$. This threshold is commonly found in the meteorological community after Isern-Fontanet et al. [13]. An extension of the Okubo-Weiss criterion to unsteady fields was proposed by Mezieć et al. [15], using the gradient of the flow map instead of $J$ directly. Note that the computation then is no longer local. While the Okubo-Weiss criterion is abundantly used, it can be shown that it is not apt to define vortex area and should thus be apploed with care $[2,4]$.

\section{Eulerian Topology-Based Eddy Detection}

Within a 2D steady flow, the boundary of an eddy is expected to be a circular stream line, closed or nearly closed [19]. A number of algorithms have been proposed to detect such eddies and trace them across time slices [22,24]. The algorithm by Petz et al. [17] utilizes lines whose tangent is not necessarily parallel to the underlying field, but incident at a constant angle. When no closed stream lines are present, nearly closed stream lines are extracted instead. Here, the winding angle method from Saderjon and Post [20] is frequently used. In case of eddies touching boundaries, as is often the case in the Red Sea, the criterion can be relaxed to accept smaller overall angles [28].

Another feature of 2D steady topology, Eulerian bifurcations, have been regarded to detect eddy formation [8]. They mark the formation of a saddle point, detaching the eddy as a new topological region.

\section{Lagrangian Topology-Based Eddy Detection}

Eddies trap mass, allowing no or little intermixing with their surroundings. They can thus be treated as topological regions even in unsteady flows, with their boundaries describing Lagrangian coherent structures (LCS). Several works approach LCS eddy boundaries using the finitetime Lyapunov exponent (FTLE) [1,3,9]. In FTLE fields, hyperbolic LCSs appear as ridge lines under forward and backward integration. An example of the forward FTLE field computed around an eddy within the Gulf of Aden is shown in Figure 2b. However, to our knowledge FTLE has not been utilized to extract the exact boundaries of eddies, but rather to investigate their splitting from other structures or their transportation. The extraction of ridges as geometry is non-trivial, and an eddy is seldom fully enclosed by FTLE structures.
Another method by Haller and Beron-Vera utilizes elliptical LCS [7] This approach finds elliptical structures that deform uniformly over time. Beron-Vera et al. [4] extract least stretching closed shear lines, detecting especially long-lived eddies.

\section{Transport-Based Eddy Detection}

One final set of algorithms are transport-based methods. When integrating particles within an eddy, they are expected to remain inside a connected region, as in Figure 3. These regions, called finite-time coherent sets, have been computed to extract extremely long-lived eddies [6].

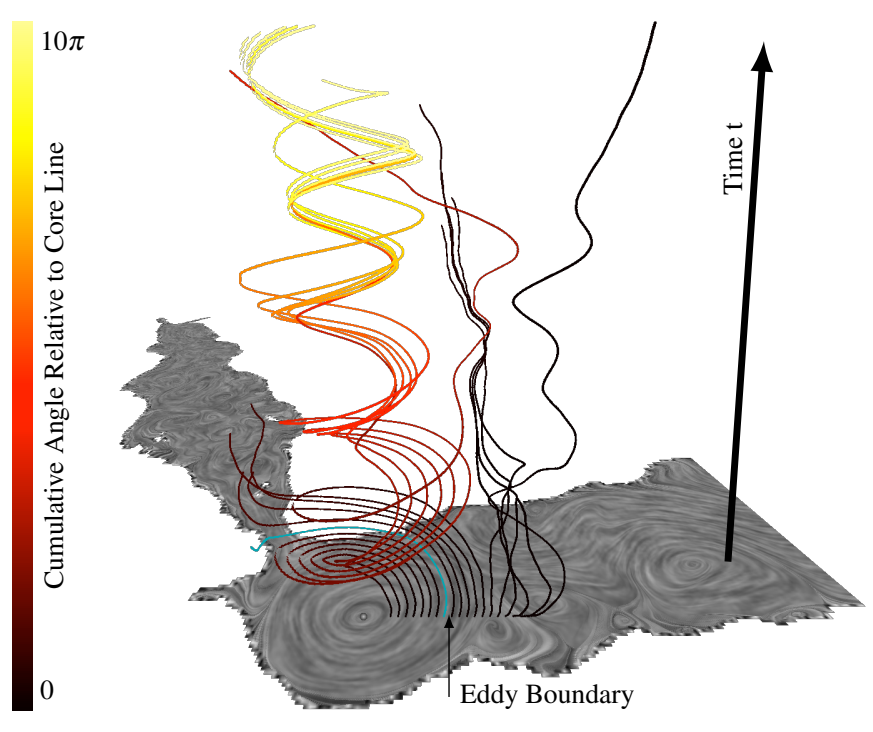

Figure 3: Example of 19 path lines moving around an eddy core. Time is displayed as height, while the angle around the common core is color-coded. All particles up to a certain distance to the core stay in a synchronous circular motion, fulfilling several turns. Further apart, paths terminate early to the right or hit the coast to the left, ending in darker colors. The path at the boundary is marked blue.

\section{Angular Distance Metric}

Eddies in the Red Sea, the Gulf of Aden and other constricted seas show some special behavior. Their coast is an important part of the eddy creation and often part of their boundary. On the other hand, the water body loses kinetic energy when interacting with the basin walls and other shearing structures induced by them. This results in smaller sizes, very irregular boundary shapes, and thus overall less stable structure.

Of the aforementioned methods, many do not apply well to this setting. Local methods do not utilize the context of the flow field, and thus rely on the Jacobian derivative, decomposed into shear and rotation components. In the Red Sea, shear and vorticity behave more erratically. The shear field of our data is illustrated in Figure 1. 


\section{Motivation}

We base our method for eddy shape extraction on a simple observation: Inside an eddy, path lines starting close together will move very similarly, revolving around a common core. They can not deviate far from each other, as they are constricted to the inside of the eddy boundary.

Outside, anything may happen in the flow. They may travel around the eddy for a certain time, but will eventually move away. An example of this is shown in Figure 3. Moving outwards from an eddy center point, we seed a small number of representative path lines. It is noteworthy that all particles staying in circular motion for the first full turn continue to do so. Thus, they are inside the eddy, all other lines are classified as outside. The eddy boundary can be defined as a point where a relatively low distance of path lines abruptly changes to a higher deviation. Another important factor visible in the example is the different speed of particles. The outmost path line within the eddy is far more elongated in time than the inner ones. It is therefor not sufficient to compare the absolute position of particles to each other. Instead, we will work in polar coordinates relative to the core line.

Several examples of distance based metrics are shown in Figure 2 on our dataset. The shear strain in Figure 2a captures distances for infinitely small integration times. The Langrangian version, the forward FTLE field, is shown in Figure 2b.

We want to use distance to find a point where a relatively stable distance between path lines changes into a more chaotic, generally expanding distance. To this end, we utilize an FTLE-like approach, making a few alterations to the original algorithm:

- Path lines are re-parametrized to polar coordinates, i.e. angle and radius. This excludes shear strain induced by particles traveling very close geometrically, but at differing angular velocities.

- We sum up the distances along the path lines instead of analyzing the end positions only. This gives the advantage of including high variations that occurred along their way. In free flow, separation is asymptotically exponential, compared to an almost constant distance within the eddy. Thus, the error measure shows a rather extreme jump across the eddy boundary.

- The deviation is measured in radial direction only, instead of looking at the maximal spread.

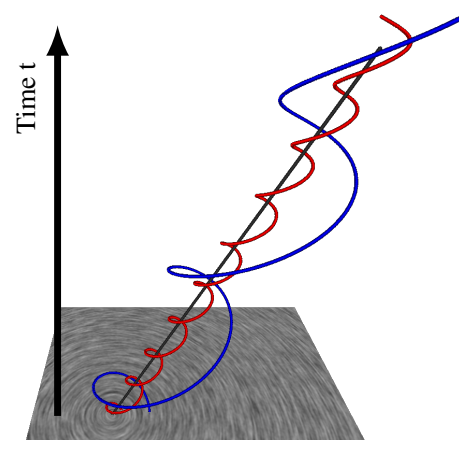

(a) Two path lines over $\tau$

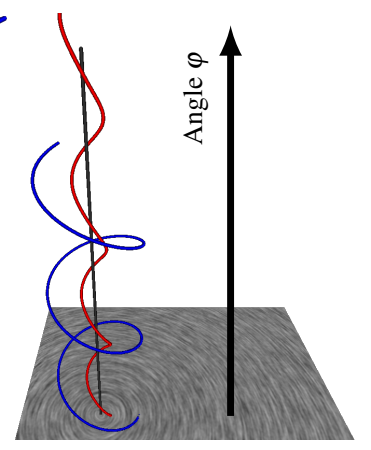

(b) Two path lines over $\varphi$
Figure 4: Two path lines in different flows around a core line (black). a) Translation over time and a change in angular velocity strongly affect the euclidean distance between particles. b) Mapping the lines to angle in relation to the core line and subtracting the translation renders the particle distance constant again.

\section{Our Approach}

Given a 2D time-dependent velocity field $\mathbf{v}(\mathbf{x}, t)$, we consider path lines within the flow field $\phi(\mathbf{x}, t, \tau)$ obeying the differential equation

$$
\frac{\partial \phi(\mathbf{x}, t, \tau)}{\partial \tau}=\mathbf{v}(\phi(\mathbf{x}, t, \tau), t+\tau) \text { and } \phi(\mathbf{x}, t, 0)=\mathbf{x} .
$$

We can compare the deviation $d$ between two path lines from seeds $\mathbf{x}_{1}$ and $\mathbf{x}_{2}$ at time $t+\tau$ by simply computing their Euclidean distance. We call the average of the distance along the line, seen in Figure 2c, path line distance: $E\left(\mathbf{x}_{1}, \mathbf{x}_{2}, t, \tau\right)=\frac{1}{\|\tau\|} \int_{0}^{s}\left\|\phi\left(\mathbf{x}_{2}, t, s\right)-\phi\left(\mathbf{x}_{1}, t, \tau\right)\right\| d s$.
In order to minimize the impact of shear flow, we apply an anglebased re-parametrization. Under a rotating flow, path lines seeded close together will only keep their distance constant if the flow exhibits a constant angular velocity. For a realistic radial profile of velocity strength, the lines will diverge (see Figure $4 a, 3$ ). This introduces a high error value contrary to the overall similar path.

We re-parametrize a point on a path line to an angle $\varphi_{c}$ and radius $r_{c}$ around a moving center point $\mathbf{c}(t)$ as in Figure $4 \mathrm{~b}$. Segments that move backwards are cut off, allowing us to define the inverse function of $\varphi_{c}$ as $\tau_{c}\left(\mathbf{x}, t, \varphi_{c}(\mathbf{x}, t, \tau)\right)=\tau$. In this reference frame, the error function is

$e_{c}\left(\mathbf{x}_{1}, \mathbf{x}_{2}, t, \alpha\right)=\frac{1}{\alpha} \int_{0}^{\alpha}\left\|r_{c}\left(\mathbf{x}_{1}, t, \tau_{c}\left(\mathbf{x}_{1}, t, \beta\right)\right)-r_{c}\left(\mathbf{x}_{2}, t, \tau_{c}\left(\mathbf{x}_{2}, t, \beta\right)\right)\right\| d \beta$.

$\alpha$ steers the maximal integration time. As we can see for example in Figure 4a, particles commonly diverge from their circular motion after no more than one full turn. Vice versa, if a path line keeps a low error distance for at least one turn, it is very likely to keep doing so. This means that an $\alpha>2 \pi$ is a sufficient integration bound.

\section{Eddy Boundary Detection}

We define our error measure

$$
E_{c}(\mathbf{x}, t, \beta)=\frac{1}{\varepsilon} e_{c}\left(\mathbf{x}, \mathbf{x}+\varepsilon \frac{\mathbf{x}-\mathbf{c}(t)}{\|\mathbf{x}-\mathbf{c}(t)\|}, t, \beta\right)
$$

i.e., comparing particles a small $\varepsilon$ apart from each other. The resulting field, shown for example in Figure 2d, has a clear area of low error inside the eddy and a sharp edge to high error values outside. This edge marks the boundary clearly, which is an important advantage over FTLE: Ridge extraction is an unsolved problem, which becomes even harder when seeking a closed boundary for area computations.

To extract a closed boundary, we utilize an active contour algorithm with balloon forces [5]. This basically means inflating a small circle around the core that tends to stop at edges, while balancing this with a maximal curvature stress. Due to the very clear edge, the result is stable under a wide range of contour parameterizations.

In Figure 2, we computed FTLE, path line distance $E$ and radial error $E_{c}$ around one eddy in the Gulf of Aden, all limited to the maximum integration length of one third of the data set. The area of the eddy is by far best defined in the $E_{c}$ field, while $E, E_{c}$ and the FTLE ridges show very similar structures. All measures would profit from longer integration times, which is unfortunately not feasible in this case. In general, our method shares most properties of FTLE. This includes limitations like the reliance on long integration times and the high computational cost, while retaining advantages like Galilean invariance.

All together, our algorithm executes as follows:

Identify the Core Lines: For each eddy, a core is traced. Several core extractors were tested, but the result was barely influenced as long as the line remains well inside the eddy (see supplemental material). For simplicity, we use a stream line core for all further computations.

Integrate Path Lines around the Core: We integrate $n$ path lines on a number of outgoing radii and evaluate the error between consecutive seed points, resulting in $n-1$ values of $E_{c}$.

Compute Error Measure: In relation to the selected core, the path lines are mapped to angle and radius. Up to a maximum angle $\alpha$, differences are integrated to obtain $E_{c}$.

Threshold Error: The boundaries of the eddy lay on the ring where the error rapidly increases. We have found the respective edges to be very sharp: While inner values commonly lay between 0 and 1 , meaning no or negative separation over time, the edges often reach values above 10 . Also, gradients are especially high there, meaning the threshold value can safely be chosen anywhere between 3 and 8 .

Extract Boundary: We grow an active contour from the center. It terminates at the edges in the thresholded error field and bridges gaps.

Connect Results of all Time Slices: The process is executed on each time slice, generating a set of closed curves. By connecting them, we gain a tubular structure in space time. We again display time in height, making the overall movement and transformation of the eddies visible, see Figure 5. The distance to the respective core line is encoded into the color of the tube, allowing to more easily identify structures. 


\section{Results}

\section{Boundaries}

We executed our algorithm on two simulations of the dataset described in subsection 1.3. The results are shown in Figure 5: In the Gulf of Aden, we can observe a very regular behavior, indicated by straight tubes dispatching from the coast line. Around the stable eddy, spiraling patterns occur. due to small cusps that slowly rotate over time.

In the simulations, we observed zero to two eddies within the Red Sea. Other vortical structures appear, but are too short-lived to be considered as eddies. In the first dataset displayed, a single eddy transforms from an elongated shape to a mostly circular one. It decays after 10 days, when highly separating ridge-like structures form in the eddy area as the structure diffuses. With neither method were we able to define a boundary from then on.

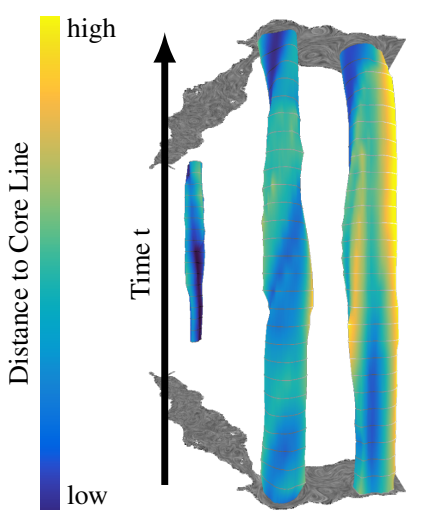

(a) Boundary tubes in simulation 0

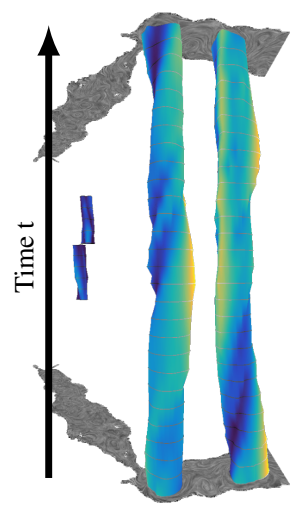

(b) Boundary tubes in simulation 3
Figure 5: Boundaries for all time steps, connected to tubes in time. The distance to the core line is color-coded on the surface. Grey lines indicate the positions in time where a boundary was computed.

To analyze the pertinence of the extracted boundaries, we compare our results to those of the Okubo-Weiss criterion, the standard in the geophysics community. The results are shown in Figure 6.

In the $E_{c}$ field, values above 3 were cut for the boundary extraction. The Okubo-Weiss field is thresholded at -0.2 the standard deviation.

In the last row, the boundaries from both methods are overlaid. For the stable eddy at the left, the areas mostly agree. Over time, the extracted boundaries are deforming only slowly, showing more rotation than deformation in the Gulf of Aden, as can be observed in Figure 5 and the accompanying video.
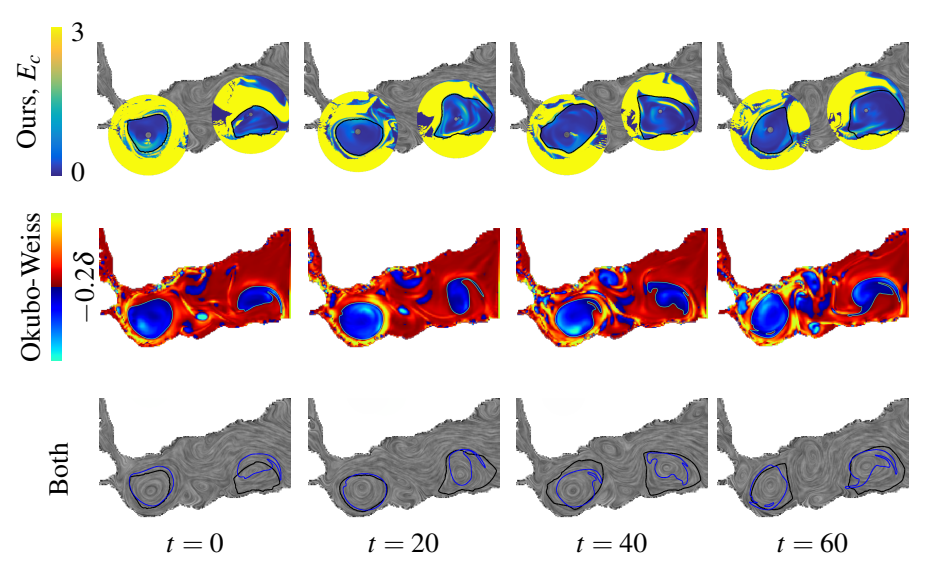

Figure 6: Boundary lines of eddies in the Gulf of Aden

First row: Error graph $E_{c}$ and extracted boundaries at 4 points in time. Second row: Okubo-Weiss field with respective boundaries.

Third row: Both results overlaid, $E_{c}$ in black, Okubo-Weiss in blue.

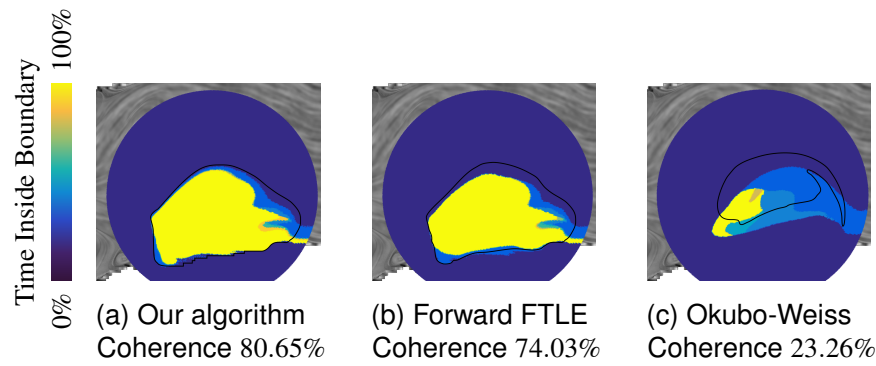

Figure 7: Coherence of the eddy tracks. The initial boundary is shown in black. We display the time that each path line leaves the boundary.

\section{Coherence}

For an estimation of the boundary quality, we compare the coherence of the extracted areas, as a defining quality of eddies is their transport of a coherent water mass over a long time. To quantify this property, we measure the time each particle spends within the eddy boundary. In a completely stable case, each particle within the eddy will remain inside indefinitely. This quantity is summarized in a single coherence value: the average time a particle from within the eddy remains in it.

We compute the coherence for our method, FTLE and Okubo-Weiss. While our method and the Okubo-Weiss criterion output an area directly, a comparison to the FTLE output is difficult. We generated the FTLE area by adapting our boundaries to coincide with the ridges, since the difference between both tracks is rather small.

The time that each particle remains within the boundary is displayed in Figure 7, with the overall average noted below. As expected, FTLE and our method perform very similarly. In contrast, the coherence achieved by the local Okubo-Weiss criterion is rather low, as expected. This confirms that the method is not suitable for our application.

\section{CONCLUSION}

\section{Summary}

Eddies in the Red Sea are difficult to extract for a number of reasons. We have developed a new Lagrangian method to determine the boundary of eddies in this environment and applied it successfully. It is based on the radial distance between path lines around a common core.

To visually encode the extracted eddies, we connected their boundaries to tubes over time in a compact visualization, depicting their deformation, movement and lifetime. Applying it on two simulation instances of the Red Sea, we have proven that we find sensible areas with both regular and highly irregular shapes. The domain expert co-authoring this paper has confirmed these findings and the overall validity of the result. Especially unexpected was the boundary movement of the eastern eddy in the Gulf of Aden: While it seems to separate from the coast early on, we have found its boundary to lay at the coast for 18 days before loosing the connection.

Within our application, we were able to extract eddy tracks that are considerably more coherent than those computed using the OkuboWeiss measure. While the resulting boundaries are very close to the FTLE ridges, our algorithm allows for an easy and automatic shape extraction that FTLE can not provide.

\section{Future Work}

While several works investigate eddy boundaries, their vertical shape and mass is an open but interesting question.

By defining the boundary through future particle behavior, we see structures from the eddy dispersal while the eddy is still coherent. To mitigate this, one could consider integrating particles not only forward in time, but also backward, since the mass remains relatively stable.

Even though our method targets these irregular eddies, it should work well on datasets of more stable eddies and can then be further compared to previous methods. Vice versa, other bays and straits such as the Gulf of Mexico are known to frequently form eddies from coastal shearing. We thus expect our algorithm to perform well in these regions.

\section{ACKNOWLEDGMENTS}

This work was supported in part by funding from King Abdullah University of Science and Technology (KAUST). 


\section{REFERENCES}

[1] F. Andrade-Canto, J. Sheinbaum, and L. Zavala Sansón. A lagrangian approach to the loop current eddy separation. Nonlinear Processes in Geophysics, 20(1):85-96, 2013.

[2] C. Basdevant and T. Philipovitch. On the validity of the "weiss criterion" in two-dimensional turbulence. Physica D: Nonlinear Phenomena, 73(12):17-30, 1994.

[3] F. J. Beron-Vera, M. J. Olascoaga, and G. J. Goni. Oceanic mesoscale eddies as revealed by lagrangian coherent structures. Geophysical Research Letters, 35(12), 2008.

[4] F. J. Beron-Vera, Y. Wang, M. J. Olascoaga, G. J. Goni, and G. Haller. Objective detection of oceanic eddies and the agulhas leakage. Journal of Physical Oceanography, 43(7):1426-1438, 2013.

[5] L. D. Cohen. On active contour models and balloons. CVGIP: Image understanding, 53(2):211-218, 1991.

[6] G. Froyland, C. Horenkamp, V. Rossi, and E. van Sebille. Studying an agulhas ring's long-term pathway and decay with finite-time coherent sets. Chaos: An Interdisciplinary Journal of Nonlinear Science, 25(8), 2015.

[7] G. Haller and F. Beron-Vera. Coherent lagrangian vortices: The black holes of turbulence. Journal of Fluid Mechanics, 731, 2013.

[8] G. Haller and A. Poje. Eddy growth and mixing in mesoscale oceanographic flows. Nonlinear Processes in Geophysics, 4:223-235, 1997.

[9] C. S. Harrison and G. A. Glatzmaier. Lagrangian coherent structures in the california current system-sensitivities and limitations. Geophysical \& Astrophysical Fluid Dynamics, 106(1):22-44, 2012.

[10] K. Hodges. A general-method for tracking analysis and its application to meteorological data. Monthly Weather Review, 122(11):2573-2586, 1994.

[11] T. Höllt, M. Hadwiger, O. Knio, and I. Hoteit. Probability Maps for the Visualization of Assimilation Ensemble Flow Data. In A. Middel, K. Rink, and G. H. Weber, editors, Workshop on Visualisation in Environmental Sciences (EnvirVis). The Eurographics Association, 2015.

[12] T. Höllt, A. Magdy, P. Zhan, G. Chen, G. Gopalakrishnan, I. Hoteit, C. D. Hansen, and M. Hadwiger. Ovis: A framework for visual analysisof ocean forecast ensembles. IEEE Transactions on Visualization and Computer Graphics, 20(8):1114-1126, 2014.

[13] J. Isern-Fontanet, E. García-Ladona, and J. Font. Vortices of the mediterranean sea: An altimetric perspective. Journal of physical oceanography, 36:87-103, 2006

[14] J. Jeong and F. Hussain. On the identification of a vortex. Journal of fluid mechanics, 285:69-94, 1995.

[15] I. Mezić, S. Loire, V. A. Fonoberov, and P. Hogan. A new mixing diagnostic and gulf oil spill movement. Science, 330(6003):486-489, 2010.

[16] A. Okubo. Horizontal dispersion of floatable particles in the vicinity of velocity singularities such as convergences. In Deep sea research and oceanographic abstracts, volume 17, pages 445-454. Elsevier, 1970.

[17] C. Petz, J. Kasten, S. Prohaska, and H.-C. Hege. Hierarchical vortex regions in swirling flow. In Computer Graphics Forum, volume 28, pages 863-870. Wiley Online Library, 2009.

[18] D. E. Raitsos, R. J. Brewin, P. Zhan, D. Dreano, Y. Pradhan, G. B. Nanninga, and I. Hoteit. Sensing coral reef connectivity pathways from space. Scientific Reports, 7(1):9338, 2017.

[19] S. K. Robinson. Coherent motions in the turbulent boundary layer. Annual Review of Fluid Mechanics, 23(1):601-639, 1991.

[20] I. A. Sadarjoen and F. H. Post. Detection, quantification, and tracking of vortices using streamline geometry. Computers \& Graphics, 24(3):333341, 2000.

[21] D. Stammer and C. Wunsch. Temporal changes in eddy energy of the oceans. Deep Sea Research Part II: Topical Studies in Oceanography, 46(1):77-108, 1999.

[22] H. Theisel, T. Weinkauf, H.-C. Hege, and H.-P. Seidel. Grid-independent detection of closed stream lines in $2 \mathrm{~d}$ vector fields. In $V M V$, volume 4 , pages 421-428, 2004.

[23] J. Weiss. The dynamics of enstrophy transfer in two-dimensional hydrodynamics. Physica D: Nonlinear Phenomena, 48:273-294, 1991.

[24] T. Wischgoll, G. Scheuermann, and H. Hagen. Tracking closed streamlines in time dependent planar flows. In VMV , pages 447-454. Citeseer, 2001.

[25] F. Yao and I. Hoteit. Thermocline regulated seasonal evolution of surface chlorophyll in the gulf of aden. PloS one, 10(3):e0119951, 2015.

[26] F. Yao, I. Hoteit, L. J. Pratt, A. S. Bower, A. Köhl, G. Gopalakrishnan, and D. Rivas. Seasonal overturning circulation in the red sea: 2. winter circulation. Journal of Geophysical Research: Oceans, 119(4):2263-2289, 2014.
[27] F. Yao, I. Hoteit, L. J. Pratt, A. S. Bower, P. Zhai, A. Köhl, and G. Gopalakrishnan. Seasonal overturning circulation in the red sea: 1 . model validation and summer circulation. Journal of Geophysical Research: Oceans, 119(4):2238-2262, 2014

[28] P. Zhan, A. C. Subramanian, F. Yao, and I. Hoteit. Eddies in the red sea: A statistical and dynamical study. Journal of Geophysical Research: Oceans, 119(6):3909-3925, 2014 